\title{
EANM/EARL FDG-PET/CT accreditation - summary results from the first 200 accredited imaging systems
}

\author{
Andres Kaalep ${ }^{1}$ (D) $\cdot$ Terez Sera $^{2,3} \cdot$ Wim Oyen $^{4} \cdot$ Bernd J. Krause $^{5} \cdot$ Arturo Chiti $^{6}$ • \\ Yan Liu ${ }^{7} \cdot$ Ronald Boellaard ${ }^{3,8,9}$
}

Received: 19 June 2017 / Accepted: 5 October 2017 /Published online: 1 December 2017

(C) The Author(s) 2017. This article is an open access publication

\begin{abstract}
Purpose From 2010 until July 2016, the EANM Research Ltd. (EARL) FDG-PET/CT accreditation program has collected over 2500 phantom datasets from approximately 200 systems and 150 imaging sites worldwide. The
\end{abstract}

Andres Kaalep and Terez Sera are contributed equally

Electronic supplementary material The online version of this article (https://doi.org/10.1007/s00259-017-3853-7) contains supplementary material, which is available to authorized users.

Andres Kaalep

kaalep@gmail.com

Ronald Boellaard

r.boellaard@umcg.nl

1 Department of Medical Technology, North Estonia Medical Centre Foundation, J. Sutiste Str 19, 13419 Tallinn, Estonia

2 Department of Nuclear Medicine, University of Szeged, Szeged, Hungary

3 On behalf of EANM Research Limited (EARL), Vienna, Austria

4 The Institute of Cancer Research, Division of Radiotherapy and Imaging, Department of Nuclear Medicine, The Royal Marsden Hospital, London, UK

5 Department of Nuclear Medicine, Rostock University Medical Center, Rostock, Germany

6 Department of Nuclear Medicine, Humanitas Clinical and Research Center, Rozzano, MI, Italy

7 European Organisation for Research and Treatment of Cancer (EORTC) Headquarters, Brussels, Belgium

8 Department of Nuclear Medicine and Molecular Imaging, University of Groningen, University Medical Center Groningen, Hanzeplein 1, Groningen, the Netherlands

9 Department of Radiology and Nuclear Medicine, VU University Medical Center, Amsterdam, The Netherlands objective of this study is to report the findings and impact of the accreditation program on the participating PET/CT systems.

Methods To obtain and maintain EARL accredited status, sites were required to complete and submit two phantom scans - calibration quality control (CalQC), using a uniform cylindrical phantom and image quality control (IQQC), using a NEMA NU2-2007 body phantom. Average volumetric SUV bias and SUV recovery coefficients (RC) were calculated and the data evaluated on the basis of quality control (QC) type, approval status, PET/CT system manufacturer and submission order.

Results SUV bias in 5\% $(n=96)$ of all CalQC submissions $(n=1816)$ exceeded $10 \%$. After corrective actions following EARL feedback, sites achieved $100 \%$ compliance within EARL specifications. $30 \%(n=1381)$ of SUVmean and $23 \%(n=1095)$ of SUVmax sphere recoveries from IQQC submissions failed to meet EARL accreditation criteria while after accreditation, failure rate decreased to $12 \%$ $(n=360)$ and 9\% $(n=254)$, respectively. Most systems demonstrated longitudinal SUV bias reproducibility within $\pm 5 \%$, while RC values remained stable and generally within $\pm 10 \%$ for the four largest and $\pm 20 \%$ for the two smallest spheres.

Conclusions Regardless of manufacturer or model, all investigated systems are able to comply with the EARL specifications. Within the EARL accreditation program, gross PET/CT calibration errors are successfully identified and longitudinal variability in PET/CT performances reduced. The program demonstrates that a harmonising accreditation procedure is feasible and achievable.

Keywords Performance $\cdot$ Harmonisation $\cdot$ PET/CT · Quantification · EARL accreditation 


\section{Introduction}

Positron emission tomography (PET) and computed tomography (CT) hybrid imaging (PET/CT) using ${ }^{18} \mathrm{~F}$-fluorodeoxyglucose (FDG) has become a routinely used and valuable tool in oncology. It is widely utilised for diagnosis, staging and restaging of various malignancies [1-12] as well as response monitoring due to its ability to measure metabolic changes [13-19]. Standard uptake value (SUV), which represents the tissue radioactivity concentration normalised to injected activity and body weight [20] is the most frequently used quantitative metric in oncology [21, 22]. Multiple factors, however, can give rise to bias [23-25] and increased variability in SUV, especially when inter-centre comparison is required from institutions lacking a uniform approach to imaging procedures [26-28]. The variability is a significant issue for clinical trials or multicentre studies utilising the quantitative potential of PET [24, 26-31]. In clinical practice, there is a wide range of PET systems installed globally including scanners developed more than 10 years ago along with brand new devices incorporating state of the art acquisition (i.e., time of flight, digital PET detectors) and reconstruction (i.e., resolution modelling) technologies [32]. In addition to various PET/CT models available, the acquisition and reconstruction parameters applied at different sites vary greatly due to local preferences [24, 32, 33]. Centres equipped with PET systems having new acquisition and reconstruction technologies available, often tend to aim for the possible best lesion detection, which may not be in line with quantitative harmonising standards [34]. The aforementioned technical factors impose a significant source of variability in PET quantification $[24,32]$ that should be addressed by the international community.

Numerous professional societies and organisations such as the Society of Nuclear Medicine and Molecular Imaging (SNMMI), American College of Radiology Imaging Network (ACRIN), Radiological Society of North America Quantitative Imaging Biomarkers Alliance (RSNA-QIBA), The American Association of Physicists in Medicine (AAPM) and the European Association of Nuclear Medicine (EANM) are promoting harmonisation of imaging procedures [35-37] in order to reduce the variability of PET image quantification in a multicentre setting. Many of these programs rely on quality control procedures utilising standard phantoms [38] for standardisation of quantification [32, 39-41] and harmonisation of PET/CT systems [35]. Review papers on describing some of the results and experience in running such programs have been published by Scheuermann et al. [39] and more recently by Sunderland et al. [32].

In 2006, the European Association of Nuclear Medicine (EANM) launched the EANM Research Ltd. (EARL) initiative. One of the main objectives of the program has been promoting multicentre nuclear medicine and research. In 2010, the FDG-PET/CT accreditation program was created in order to address variability in the quickly growing field of quantitative FDG-PET imaging by setting up guidelines and specifications to which the participating sites must adhere. The bandwidths for the current EARL specifications were developed during a pilot study in 2010-2011 involving 12 PET/CT systems. Based on this study, specifications for SUVmean and SUVmax recovery coefficients were derived, which accommodated all investigated systems. From its initiation until July 2016, EARL has collected approximately 2500 phantom datasets from more than $200 \mathrm{PET} / \mathrm{CT}$ systems from over 150 imaging sites worldwide. The data analysed by EARL encompasses the majority of the system types in clinical use over the past 10 years and incorporates sites with various backgrounds giving it a broad basis to represent the field as a whole.

The objective of this paper is to report the findings obtained so far in the EARL standardisation program and their impact on the quantitative variability of accredited PET/CT systems. Analysis of phantom scans from the largest number of active PET centres so far provides representative details of current quantitative capabilities of FDG-PET imaging and the variability to be expected. Understanding the characteristics of variability and the impact on standardisation will help planning multi-centre clinical trials, utilising quantitative FDGPET/CT imaging and advance use of PET as a quantitative imaging biomarker.

The secondary objective of this study is to explore ways to improve the EARL FDG-PET accreditation program based on the retrospective analysis of phantom data collected in the EARL database.

\section{Materials and methods}

\section{Acquisition and submission of data to EARL}

Sites, which are seeking EARL FDG-PET/CT accreditation for the first time, need to pass the initial procedure. This procedure includes the submission of an online questionnaire and a signed statement - these documents have to be submitted at the start of the accreditation procedure and revised annually, whereas QC documents need to be regularly provided in order to maintain the EARL accredited status.

For the first and follow-up procedures, sites have to perform calibration QC and image quality QC measurements. The calibration QC measurements have to be repeated every 3 months and image quality QC procedures annually, while the data needs to be provided to EARL upon completion of the procedures. During each round of QC survey, there is a 3 week period for the sites to collect the data and submit it to EARL, followed by a 3 week period of analysing the data by EARL and reporting the results back to the sites.

For the calibration QC measurements, centres are asked to use a cylindrical phantom with the following characteristics: 
diameter of about $20 \mathrm{~cm}$ ( 17 to $22 \mathrm{~cm})$ and length sufficient to cover the entire axial field of view (FOV). Furthermore, the exact volume of the calibration phantom should be known and recorded in the calibration QC scan report form. The phantom has to be filled with water and about $70 \mathrm{MBq}{ }^{18} \mathrm{~F}-\mathrm{FDG}$ added to it, aimed at expected phantom acquisition time.

For image quality QC measurements, the NEMA NU22007 image quality phantom is required. The phantom has a fillable torso cavity to act as a background compartment, a $5 \mathrm{~cm}$ diameter cylindrical lung insert in the centre and six fillable spheres with internal diameters of $10 \mathrm{~mm}, 13 \mathrm{~mm}$, $17 \mathrm{~mm}, 22 \mathrm{~mm}, 28 \mathrm{~mm}$ and $37 \mathrm{~mm}$ positioned coaxially around the lung insert. The phantom background compartment and the spherical inserts have to be filled with ${ }^{18} \mathrm{~F}-$ FDG solution aimed at activity concentrations at the start of the PET scan of $2 \mathrm{kBq} / \mathrm{mL}$ and $20 \mathrm{kBq} / \mathrm{mL}$, respectively, resulting in a sphere to background ratio of 10:1.

With both phantoms, routine quantitative whole body PET/ CT scans have to be performed with two PET bed positions of at least 5 min each, including a (low dose) CT for attenuation correction purposes [35]. After reconstruction, the attenuation corrected PET, non-attenuation corrected PET and CT images of the phantoms have to be uploaded into the EARL central database, along with scan report forms.

\section{Quantitative analysis and approval by EARL}

The uniform calibration QC phantom and NEMA NU2 IQ body phantom images uploaded into the EARL database are evaluated centrally, making use of a standardised semiautomatic quantitative analysis tool developed internally within EARL. The software uses activity and time information provided by the scan report forms. The average volumetric SUV bias is generated as relative deviation between measured and calculated activity concentration values (Eq. 1). The SUV recovery coefficients (RCs) for the six spherical inserts are based on $50 \%$ background corrected isocontour VOI $\left(\mathrm{RC}_{\mathrm{SUVmean}}\right)$ and maximum voxel value included in the VOI $\left(\mathrm{RC}_{\text {SUVmax }}\right)$.

SUVbias $(\%)=\left(\frac{C_{\text {measured }}}{C_{\text {calculated }}}-1\right) \times 100 \%$;

, where

$C_{\text {measured }}$-activity concentration measured from images

$C_{\text {calculated }}$-activity concentration calculated from injection data

EARL is applying SUV bias and RC values acceptance criteria, which were defined by feasibility studies performed on the systems used in clinical practices at the start of the standardisation - a study is underway in order to update these. When approval is not granted, the site undergoing (re-)accreditation is asked to take corrective actions, for example: recalibration of the PET system, adjustment of reconstruction parameters, repeating the phantom scan and so on. When required, EARL is advising the sites. A Manual describing the accreditation program in detail as well as information on the EARL website [42] is also available. If submitted QC documents meet the standard requirements, FDG-PET/CT accreditation is granted, and the department is listed on the EARL website (http://earl.eanm.org) as an accredited PET/CT centre of excellence. Furthermore, the site is provided with an accreditation certificate and signet, which can be used on its correspondence and website.

\section{Data clean-up and preparation}

To allow for data extraction, the EARL database had to be cleaned of duplicates and entries with insufficient or missing information removed, entry errors were identified and the individual site identification data ignored thereby providing an anonymised set of data for evaluation. First and subsequent site submissions were identified and marked as such.

\section{Analysis}

The calibration QC and image quality QC datasets from the EARL database will be analysed based on the type of the phantom, accreditation approval status, manufacturer of the PET/CT system and whether it was the first or a subsequent QC data submission. The SUV bias and normalised SUV biases were analysed as well as the recovery coefficients for each sphere size, separately for SUVmean and SUVmax. For each parameter, mean, median, standard deviation, standard error and skewness were calculated. Longitudinal reproducibility analysis was performed on 16 systems (systems A to P) selected based on each having sufficient longitudinal data of at least 18 approved CalQC data submissions or at least five approved IQQC datasets.

\section{Results}

\section{General overview}

Data reviewed in this paper encompasses all submissions to the EARL database from the initiation of the standardisation program in November 2010 to July 2016. Figure 1 represents the number of sites and systems participating each year. After correcting for erroneous, partial and duplicate entries, 1816 CalQC and 778 IQQC datasets were used for further analysis. The datasets were 29\% ( $n=752)$ from GE-, 29\% $(n=741)$ from Philips- and 42\% ( $n=1101)$ from Siemens-systems.

First data submissions constitute $10 \%(n=175)$ of all CalQC and 23\% $(n=178)$ of all IQQC scans. $85 \%$ 
Fig. 1 Number of sites and PET/ CT systems participating in the EARL accreditation program. For 2016 data has been collected from January to July

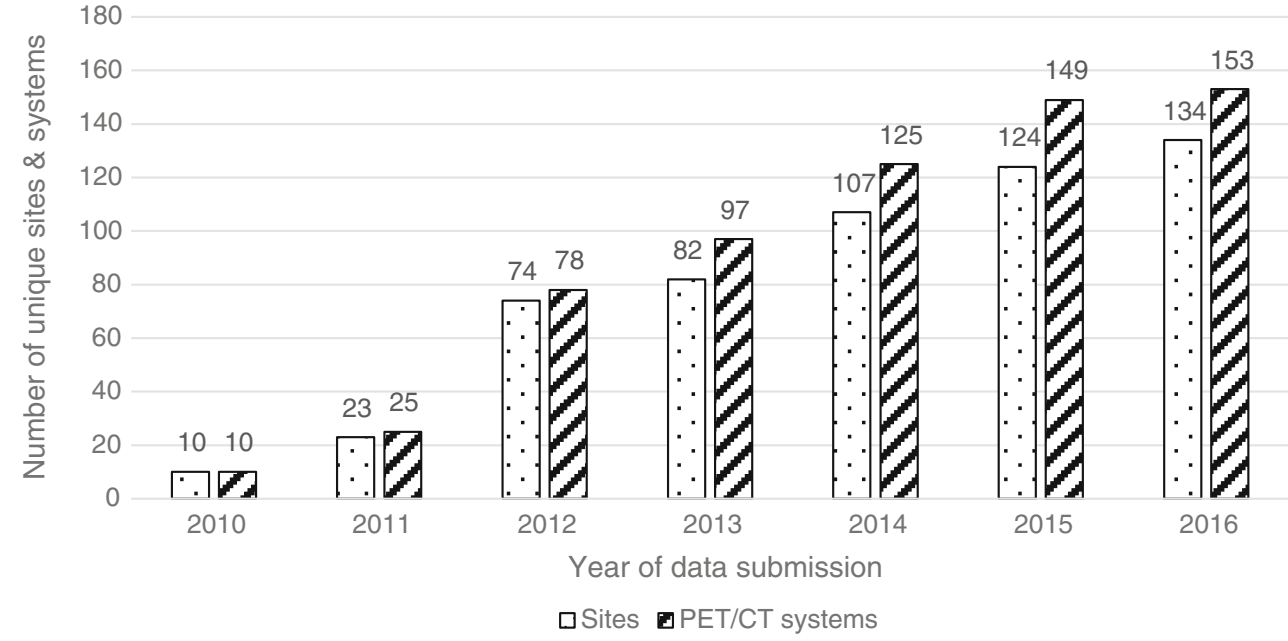

( $n=149$ ) of the first and 94\% ( $n=1537)$ of subsequent CalQC data submissions could be approved by EARL. This results in an overall approval rate for CalQC of $93 \%(n=1686)$. Table 1 states descriptive statistics for CalQC initial and subsequent submissions.

Out of all systems $(n=200)$ that have enrolled in the program, the accreditation for 47 systems (24\%) has been discontinued for various reasons, such as scanner replacement or stopped participation in trials requiring EARL accreditation.

\section{Calibration QC}

Detailed descriptive statistics for CalQC SUV bias are summarised in Table 1. Figure 2 demonstrates CalQC SUV bias distribution for all, initial and subsequent submissions along with vendor based distribution of approved results. It was found 3\% $(n=60)$ of all CalQC submissions were below and $2 \%(n=36)$ above the corresponding EARL SUV bias limits of $-10 \%$ and $+10 \%$. Also, $9 \%(n=16)$ of systems could not be approved at first CalQC submission, but after corrective actions all of the scanners fulfilled the EARL specifications. Significant mean SUV biases of $-1.53 \%(p<0.0001)$ and $-1.78 \%(\mathrm{p}<0.0001)$ were observed in approved datasets from GE and Philips systems, respectively, while datasets from Siemens systems did not demonstrate this deviation. In Fig. 3 longitudinal CalQC volumetric SUV bias is plotted as a function of the order of subsequent submissions.

\section{Image quality QC}

Descriptive statistics for IQQC SUVmax and IQQC SUVmean results for each sphere size are summarised in Supplemental Tables 1 and 2. Figure 4 shows the distribution of sphere recoveries of all submitted data and approved IQQC submissions along with SUV recovery distributions separately per manufacturer. A large variability in sphere recoveries was observed. Out of all sphere recoveries from IQQC datasets,
$30 \%(n=1381)$ of SUVmean and $23 \%(n=1095)$ of SUVmax recoveries failed to meet the EARL accreditation criteria. After corrective actions the corresponding values dropped to $12 \%(n=360)$ and $9 \%(n=254)$ respectively. A positive bias in the recovery values was observed prior to and also after the corrective actions and granted accreditation, respectively. $47 \%$ $(n=84)$ of sites' initial IQQC submissions and 68\% $(n=409)$ of all consecutive IQQC submissions were approved by EARL. Overall approval rate for IQQC submissions was $63 \%(n=493)$.

To evaluate the longitudinal performance of the 16 systems, sphere recoveries for all sphere sizes were plotted based on the order of subsequent submissions, as seen in Fig. 5. From the figure, it can be seen that for each sphere there is an initial large variability in observed SUV recoveries, which is reduced and becomes harmonised during subsequent submissions.

\section{Discussion}

The number of sites participating in the EARL accreditation program is steadily increasing and the received data is almost equally distributed among the three major PET/CT system manufacturers.

Inconsistency was observed in the names provided for same types of systems and in some cases the device serial numbers were missing or had been changed at the occasion of software upgrade or service maintenance. This complicated the distinction between new systems and those already existing in the EARL database. As a solution, the EARL database client portal could be configured so that the system type selection be performed by choosing from a predefined list, in the same way as it currently works for system manufacturer, permitting that the regularly submitted QC data be checked and, if necessary, corrected for constancy of the core data and the device serial number in particular. 
Table 1 CalQC SUV bias statistics from first, regular ongoing and all EARL approved submissions (pooled and per vendor)

\begin{tabular}{|c|c|c|c|c|c|c|c|}
\hline CalQC & $\begin{array}{l}\text { Mean SUV } \\
\text { bias }(\%)\end{array}$ & $\begin{array}{l}\text { Median } \\
\text { SUV } \\
\text { bias }(\%)\end{array}$ & $\begin{array}{l}\text { SUV bias } \\
\text { Std. Dev } \\
(\%)\end{array}$ & Skewness & $\begin{array}{l}\text { Submissions } \\
\text { with SUV } \\
\text { bias below } \\
\text { EARL specs }\end{array}$ & $\begin{array}{l}\text { Submissions } \\
\text { with SUV } \\
\text { bias above } \\
\text { EARL specs }\end{array}$ & $\begin{array}{l}\text { Submissions } \\
\text { with SUV } \\
\text { bias within } \\
\text { EARL specs }\end{array}$ \\
\hline All CalQC & $-1.14( \pm 0.13)$ & -1.01 & 5.36 & -0.32 & $3 \%$ & $2 \%$ & $95 \%$ \\
\hline All approved CalQC & $-0.97( \pm 0.09)$ & -0.94 & 3.71 & 0.15 & $0 \%$ & $0 \%$ & $100 \%$ \\
\hline Sites' first submitted CalQC & $-1.25( \pm 0.46)$ & -0.79 & 6.06 & -1.14 & $6 \%$ & $3 \%$ & $91 \%$ \\
\hline $\begin{array}{l}\text { Subsequent approved } \\
\text { submissions CalQC }\end{array}$ & $-1.01( \pm 0.09)$ & -1.02 & 3.66 & 0.16 & $0 \%$ & $0 \%$ & $100 \%$ \\
\hline All approved GE CalQC & $-1.53( \pm 0.15)$ & -1.60 & 3.27 & 0.31 & $0 \%$ & $0 \%$ & $100 \%$ \\
\hline All approved Philips CalQC & $-1.78( \pm 0.18)$ & -1.71 & 3.89 & 0.26 & $0 \%$ & $0 \%$ & $100 \%$ \\
\hline $\begin{array}{l}\text { All approved Siemens } \\
\text { CalQC }\end{array}$ & $-0.05( \pm 0.14)$ & 0.06 & 3.65 & 0.01 & $0 \%$ & $0 \%$ & $100 \%$ \\
\hline
\end{tabular}

A small number $(n=19)$ of calibration QC submissions were not included in further analysis due to large SUV biases observed. The high values might have been related to improper data communication from the participants, inhomogeneous phantoms or system failures left unnoticed by the centres. In all these cases, sites were asked to implement corrective actions and redo the experiments. As a result, all of the affected systems achieved compliance with the EARL specifications and were granted accreditation.
Regardless of manufacturer or model, all systems were able to comply with the CalQC specifications set up by EARL. Only 9\% of sites first QC submissions and 5\% of all CalQC submissions demonstrated a measured activity bias of more than $10 \%$. Scheuermann et al. in their review of ACRIN PET Core Laboratory program reported a similar initial SUV or normalisation calibration failure rate of $12 \%$ within the same acceptance criteria of SUV $1.0 \pm 0.1[39]$.

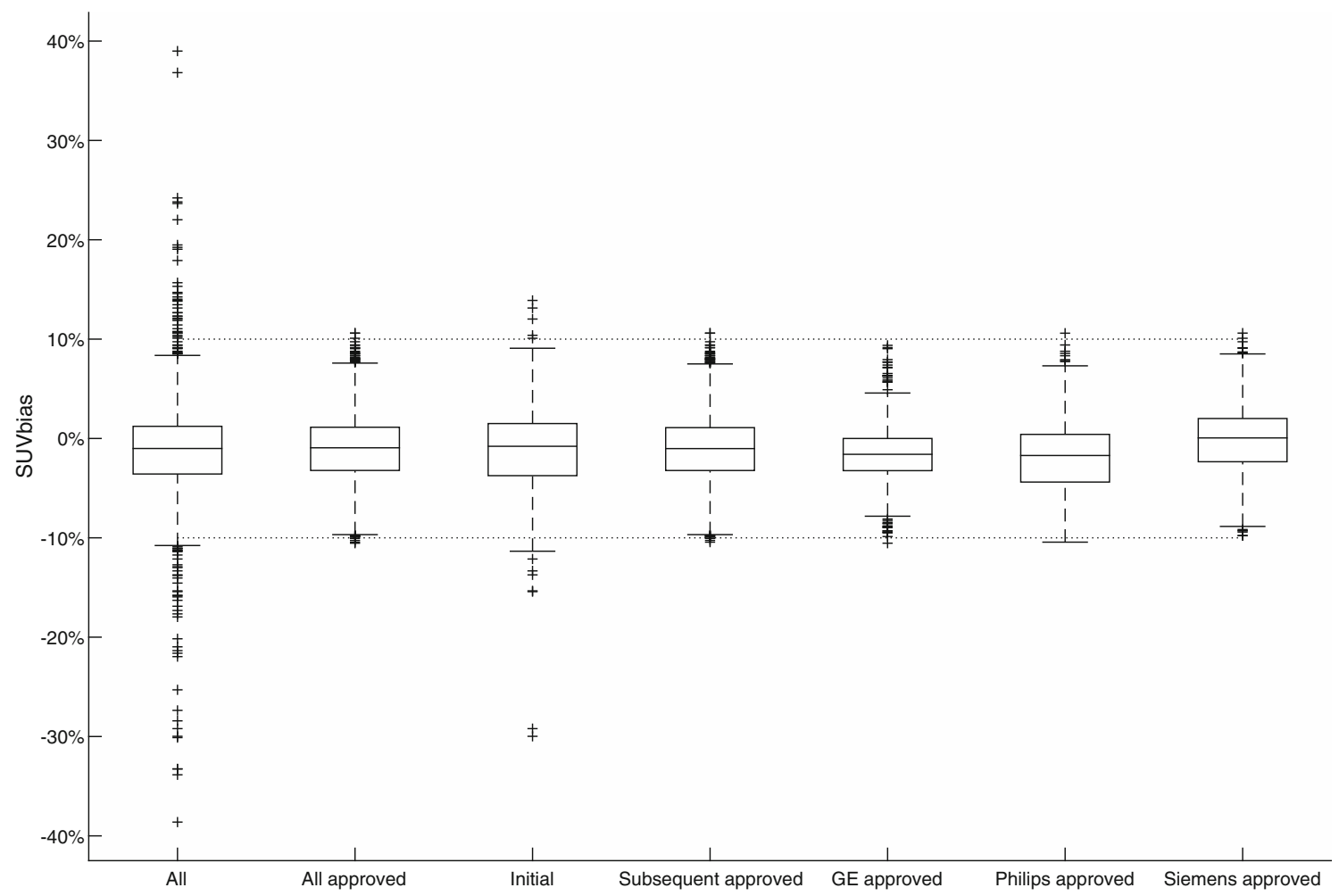

Fig. 2 Comparison of CalQC SUV bias distribution for all, initial and subsequent submissions along with vendor based distribution of approved results. The dotted horizontal lines represent EARL specification limits. Central line of the box is the median, edges of the

box are the 25th and 75th percentiles, the whiskers extend to either of the most extreme data points, which are not considered outliers or 1.5 times interquartile range. The outliers are marked using plus signs 


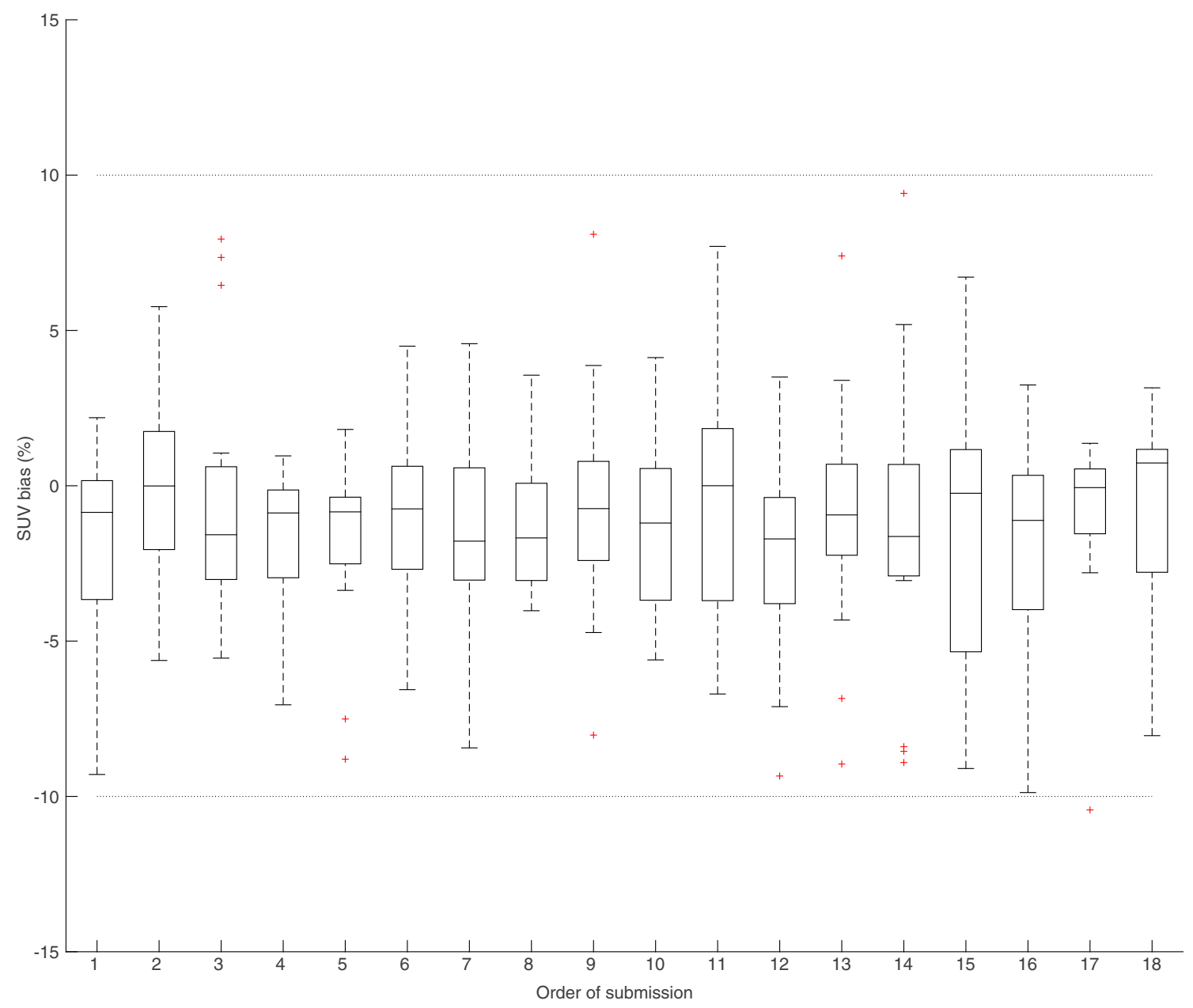

Fig. 3 Longitudinal plots of EARL approved CalQC results from the 16 systems. SUV bias deviation from the expected value. The dotted horizontal lines represent EARL specification limits. Central line of the box is the median, edges of the box are the 25th and 75th percentiles, the whiskers extend to either of the most extreme data points, which are not considered outliers or 1.5 times interquartile range. The outliers are marked using plus signs

note that these errors were well within $2 \%$ and are very likely to be clinically irrelevant.

Longitudinal analysis of the CalQC results from 16 selected systems, visualised in Fig. 6, illustrates good performance of the systems and reflects the quantification stability achieved by the accredited sites. Large SUV biases were only occasionally observed, and were all corrected by the sites after receiving notification from EARL. In most of the cases, corrective action had been taken within 4 weeks.

The majority of PET/CT systems followed throughout the investigation period showed good reproducibility of the CalQC results seen in Fig. 3. Longitudinal SUV bias values lie mostly within $\pm 5 \%$ of the expected value, which is in agreement with data published by Geworski et al. [43] and more recently by Sunderland et al. [32]. The rest of the systems also meet the $\pm 10 \%$ calibration accuracy criteria. Our findings suggest opportunities for the implementation of stricter accreditation specification for EARL CalQC SUV biases. 

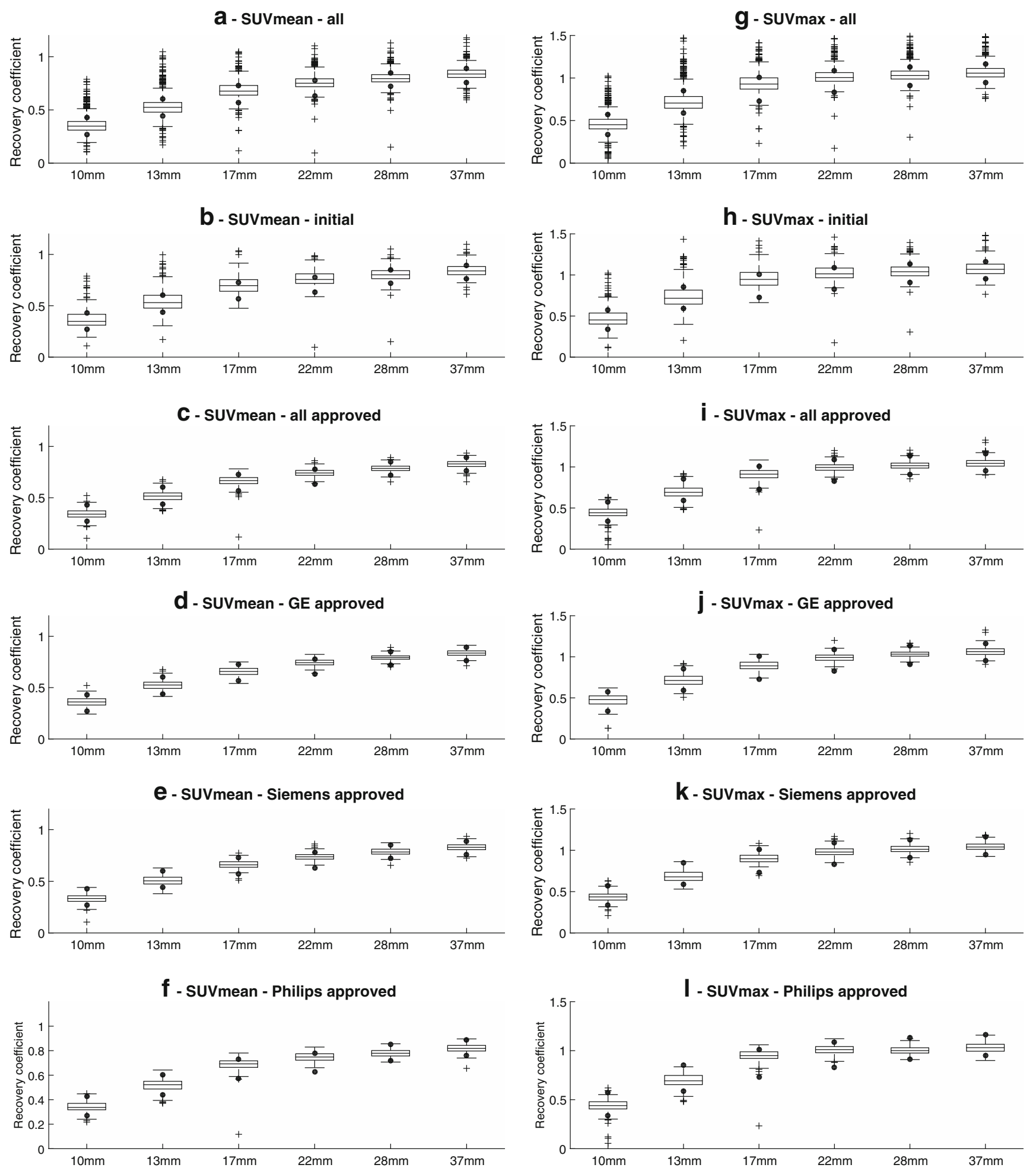

Fig. 4 IQQC SUVmean (a to f) and SUVmax (g to l) recovery results, regular ongoing and all EARL approved submissions (pooled and per vendor). Dots represent EARL specification limits. Central line of the box is the median, edges of the box are the 25 th and 75 th percentiles,

The comparison between all received and EARL approved data indicates significant reduction of outliers for IQQC results (Supplemental Tables 1 and 2). However, some of the data

the whiskers extend to either of the most extreme data points which are not considered outliers or 1.5 times interquartile range. The outliers are marked using plus signs

not strictly within EARL specifications was still accepted after critical review. This means the noncompliant data was deemed acceptable in case only one of the spheres being slightly out of 

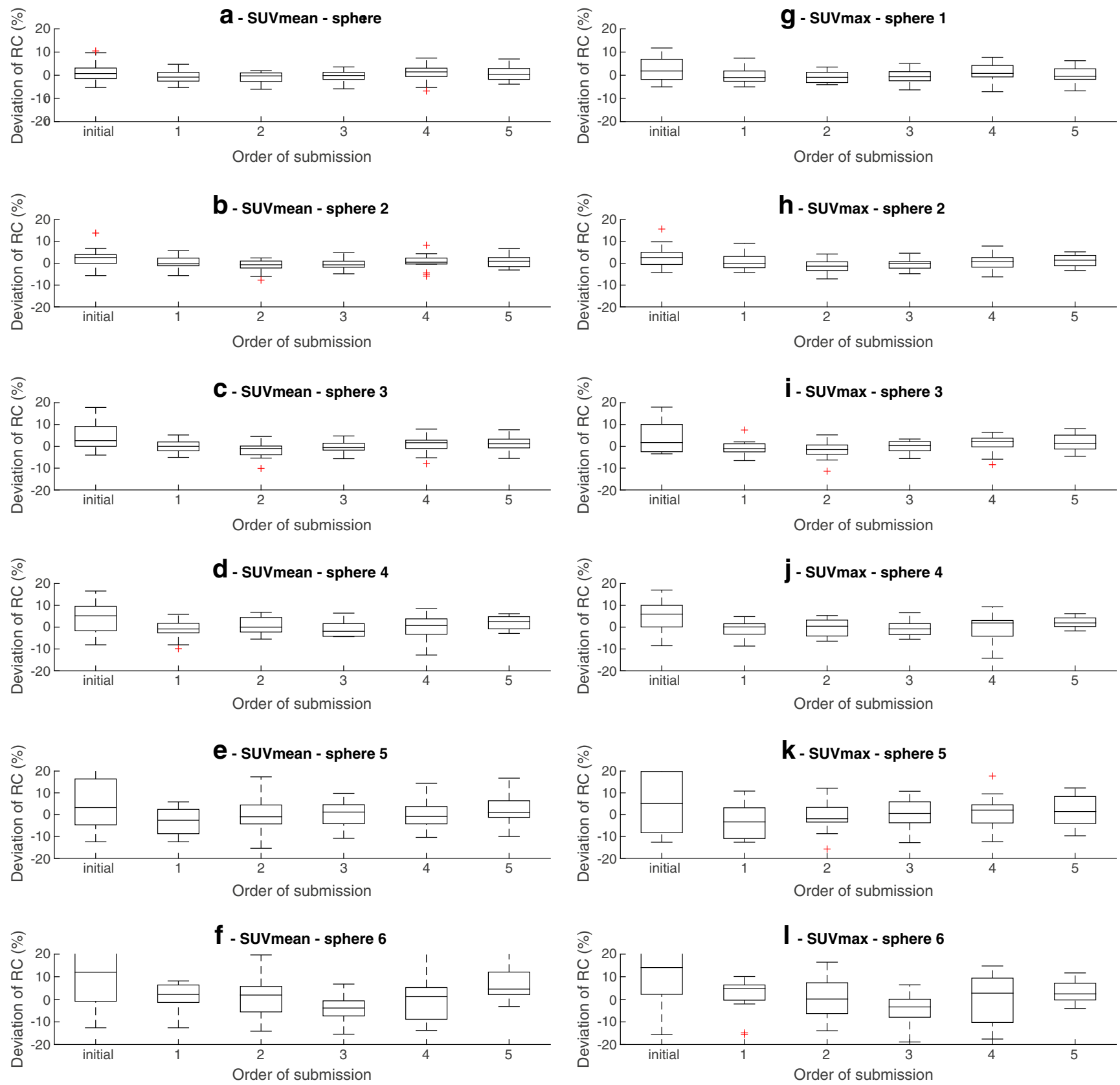

Fig. 5 Longitudinal analysis of IQQC results from 16 scanners. Recovery coefficients biases from the mean of the respective scanner. "Initial"data series represents the first IQQC submission for each system. Central line of the box is the median, edges of the box are the

the specifications, but the overall shape and magnitude of RC curve could be determined to a sufficient extent and being compliant with EARL criteria. Moreover, in these cases submissions were reviewed retrospectively and consistency of acquisition, reconstruction and settings was verified. While in case of the larger diameter spheres meeting EARL specifications was common, for the smallest sphere (10 $\mathrm{mm}$ diameter) SUVmax data remained outside of specified bandwidth in $26 \%$ of the EARL approved submissions. Achieving harmonised

25th and 75th percentiles, the whiskers extend to either of the most extreme data points which are not considered outliers or 1.5 times interquartile range. The outliers are marked using plus signs

quantitative results for the smallest sphere turned out to be challenging due to its increased sensitivity to image noise and phantom positioning within the PET field of view.

Although in most cases when the spheres' RCs did not meet the specifications, sites were asked to redo experiments, still a noticeable upward bias in results for the smallest sphere could be observed. Therefore, based on these findings EARL implemented slightly adjusted recovery specifications for the smallest sphere starting from the 1st of January 2017. 
Fig. 6 Longitudinal analysis of the 16 systems' CalQC results. SUV bias values for each system are presented as separate lines. Dotted lines represent outliers and dashed lines subsequent corrective actions. Red dots represent data points outside EARL specifications

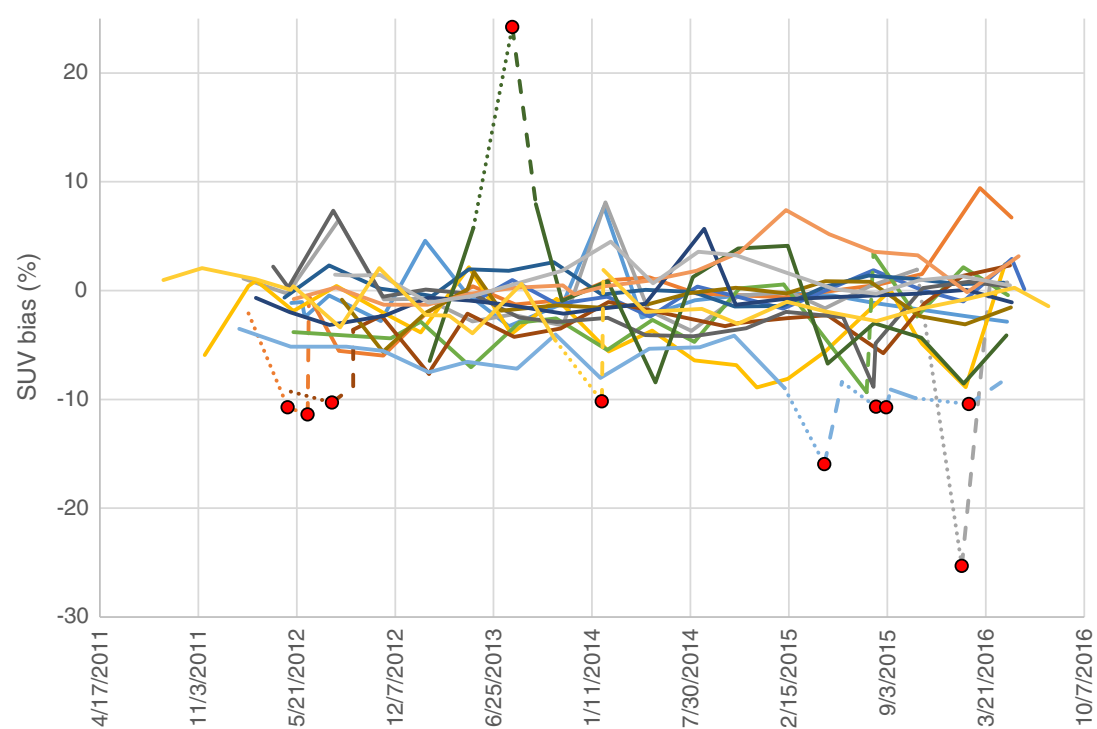

Prior to harmonisation, the average recoveries of all spheres demonstrated a positive bias compared to EARL specifications. The trend could still be observed to some extent within the approved results. This can be attributed to both the vendors and sites preferences leaning towards reconstruction algorithms emphasising contrast and detectability. The positive bias in the case of the $10 \mathrm{~mm}$ diameter sphere might also be attributed to newly adopted acquisition and reconstruction technologies (e.g., including resolution modelling), which aims at increasing small lesion detectability. This trend was also observed by Sunderland et al. who showed that recoveries were generally higher for systems employing TOF and PSF reconstructions [32]. Since more modern PET/CT systems with new technologies appear in the field, a review of the existing EARL specifications is required in order to facilitate the inclusion of the increased contrast recovery capabilities of these systems. EARL is currently carrying out a feasibility study aiming at redefining the accreditation criteria by taking into account not only the new technologies but also considering that the majority of the PET/CT systems currently in clinical use should be able to comply.

By comparing the first and following regular submissions from participating sites, a relative increase can be observed in meeting the EARL specifications which is expected since the accredited sites gain experience in performing quantitative calibrations and assessment of their systems. The staff's increased awareness towards the necessity of regular calibration and quality control of the systems is one of the benefits of participation in an accreditation/standardisation program, which is difficult to measure but should not be underestimated.

IQQC longitudinal analysis results, presented in Fig. 5 visualise the major improvement of regular submissions in relation to the first submission. As the sphere size decreases, the improvement becomes more prominent. After initial adjustment of the reconstruction parameters, the longitudinal reproducibility remains stable and generally within $\pm 10 \%$ for the largest four spheres and $\pm 20 \%$ for the smallest two spheres. These findings emphasise that PET/CT performance and the execution of the QC experiments show high reproducibility and demonstrate that long-term maintenance of a harmonised PET/CT network is feasible and achievable.

\section{Conclusion}

The European Association of Nuclear Medicine (EANM) has been running an FDG-PET/CT accreditation program under the EANM Research Ltd. (EARL) initiative in order to harmonise quantitative PET/CT performance and facilitate multicentre nuclear medicine and research. The number of sites and systems participating in the accreditation program has steadily increased over the years with similar numbers of scanners from each of the three major vendors.

Outliers observed in the overall submissions of both CalQC and IQQC were largely eliminated in subsequent submissions after notification from EARL. Excellent longitudinal performance was observed in most of the systems - a majority demonstrated CalQC values reproducible within $5 \%$ and IQQC results within $10 \%$ for the largest four spheres and $20 \%$ for the smallest two spheres. Occasional deviations from expected values were rapidly resolved by the sites after notification from EARL. Regardless of system manufacturer or model, all vendors were able to comply with the accreditation specifications set out by EARL.

Prior to harmonisation, IQQC data demonstrated a slight positive bias relative to EARL specifications, which suggested carrying out a review and an update in order to account for the advances in acquisition and reconstruction technologies in PET/CT. 
In this manuscript we have demonstrated that the EARL accreditation program can successfully identify gross PET/CT calibration errors and reduces variability in PET/CT performance by longitudinally performing harmonisation QC experiments. The program is running successfully for more than 6 years and shows that a harmonising accreditation procedure is feasible and achievable.

Centres with accredited PET/CT systems benefit greatly from participating in large-scale accreditation programs, which facilitate the implementation of procedural guidelines widely recognised by the imaging community.

Acknowledgements The publication of this article was supported by funds of the European Association of Nuclear Medicine (EANM).

Compliance with ethical standards Andres Kaalep declares that he has no conflict of interest. Terez Sera has received travel grants and honoraria from EARL. Wim Oyen declares that he has no conflict of interest. Bernd J. Krause declares that he has no conflict of interest. Yan Liu declares that she has no conflict of interest. Arturo Chiti has received travel grants and speaker honoraria from General Electric Medical Systems. Ronald Boellaard is a member of the EARL scientific advisory board.

This article does not contain any studies with human participants or animals performed by any of the authors.

Open Access This article is distributed under the terms of the Creative Commons Attribution 4.0 International License (http:// creativecommons.org/licenses/by/4.0/), which permits unrestricted use, distribution, and reproduction in any medium, provided you give appropriate credit to the original author(s) and the source, provide a link to the Creative Commons license, and indicate if changes were made.

\section{References}

1. Fletcher JW, Djulbegovic B, Soares HP, Siegel BA, Lowe VJ, Lyman GH, et al. Recommendations on the use of 18F-FDG PET in oncology. J Nucl Med. 2008;49:480-508.

2. de Geus-Oei L-F, van der Heijden HFM, Corstens FHM, Oyen WJG. Predictive and prognostic value of FDG-PET in nonsmallcell lung cancer: a systematic review. Cancer. 2007;110:1654-64.

3. Shankar LK, Hoffman JM, Bacharach S, Graham MM, Karp J, Lammertsma AA, et al. Consensus recommendations for the use of $18 \mathrm{~F}-F D G$ PET as an indicator of therapeutic response in patients in National Cancer Institute trials. J Nucl Med. 2006;47:1059-66.

4. Gupta T, Master Z, Kannan S, Agarwal JP, Ghsoh-Laskar S, Rangarajan V, et al. Diagnostic performance of post-treatment FDG PET or FDG PET/CT imaging in head and neck cancer: a systematic review and meta-analysis. Eur J Nucl Med Mol Imaging. 2011;38:2083.

5. Ung YC, Maziak DE, Vanderveen JA, Smith CA, Gulenchyn K, Lacchetti C, et al. 18Fluorodeoxyglucose positron emission tomography in the diagnosis and staging of lung cancer: a systematic review. J Natl Cancer Inst. 2007;99:1753-67.

6. Lv Y-L, Yuan D-M, Wang K, Miao X-H, Qian Q, Wei S-Z, et al. Diagnostic performance of integrated positron emission tomography/computed tomography for mediastinal lymph node staging in non-small cell lung cancer: a bivariate systematic review and meta-analysis. J. Thorac. Oncol. International Association for the Study of. Lung Cancer. 2011;6:1350-8.
7. Eschmann SM, Friedel G, Paulsen F, Reimold M, Hehr T, Budach W, et al. 18F-FDG PET for assessment of therapy response and preoperative re-evaluation after neoadjuvant radio-chemotherapy in stage III non-small cell lung cancer. Eur J Nucl Med Mol Imaging. 2007;34:463-71.

8. Hicks RJ, Kalff V, Macmanus MP, Ware RE, Mckenzie AF, Matthews JP, et al. The utility of F-FDG PET for suspected recurrent non - small cell lung cancer after potentially curative Therapy : impact on management and prognostic stratification. J Nucl Med. 2001:42:10-21.

9. Dijkman BG, Schuurbiers OCJ, Vriens D, Looijen-Salamon M, Bussink J, Timmer-Bonte JNH, et al. The role of (18)F-FDG PET in the differentiation between lung metastases and synchronous second primary lung tumours. Eur J Nucl Med Mol Imaging. Berlin/Heidelberg: Springer-Verlag. 2010;37:2037-47.

10. Hellwig D, Graeter TP, Ukena D, Groeschel A, Sybrecht GW, Schaefers HJ, et al. 18F-FDG PET for mediastinal staging of lung cancer: which SUV threshold makes sense? J Nucl Med. 2007;48: 1761-6.

11. Agarwal M, Brahmanday G, Bajaj SK, Ravikrishnan KP, Wong CYO. Revisiting the prognostic value of preoperative (18)F-fluoro-2deoxyglucose ( (18)F-FDG) positron emission tomography (PET) in early-stage (I \& II) non-small cell lung cancers (NSCLC). Eur J Nucl Med Mol Imaging. 2010;37:691-8.

12. Liao S, Penney BC, Wroblewski K, Zhang H, Simon CA, Kampalath R, et al. Prognostic value of metabolic tumor burden on 18F-FDG PET in nonsurgical patients with non-small cell lung cancer. Eur J Nucl Med Mol Imaging. 2012;39:27-38.

13. Weber WA. Use of PET for monitoring cancer therapy and for predicting outcome. J Nucl Med. 2005;46:983-95.

14. Hicks RJ. Role of 18 F-FDG PET in assessment of response in nonsmall cell lung cancer. J Nucl Med. 2009;50:31S-42.

15. Czernin J, Weber WA, Herschman HR. Molecular imaging in the development of cancer therapeutics. Annu Rev Med United States. 2006;57:99-118.

16. Frank R, Hargreaves R. Clinical biomarkers in drug discovery and development. Nat Rev Drug Discov England. 2003;2:566-80.

17. Weber WA. Assessing tumor response to therapy. J Nucl Med. 2009;50:1S-0.

18. Bengtsson T, Hicks RJ, Peterson A, Port RE. 18F-FDG PET as a surrogate biomarker in non-small cell lung cancer treated with erlotinib: newly identified lesions are more informative than standardized uptake value. J Nucl Med. 2012;53:530-7.

19. Huang W, Zhou T, Ma L, Sun H, Gong H, Wang J, et al. Standard uptake value and metabolic tumor volume of $18 \mathrm{~F}-\mathrm{FDG}$ PET/CT predict short-term outcome early in the course of chemoradiotherapy in advanced non-small cell lung cancer. Eur J Nucl Med Mol Imaging. 2011;38:1628.

20. Thie JA. Understanding the standardized uptake value, its methods, and implications for usage. J Nucl Med. 2004;45:1431-4.

21. Weber WA, Petersen V, Schmidt B, Tyndale-Hines L, Link T, Peschel C, et al. Positron emission tomography in non-small-cell lung cancer: prediction of response to chemotherapy by quantitative assessment of glucose use. J Clin Oncol United States. 2003;21: 2651-7.

22. Kelly MD, Declerck JM. SUVref: reducing reconstructiondependent variation in PET SUV. EJNMMI Res. 2011;1:16.

23. Adams MC, Turkington TG, Wilson JM, Wong TZ. A systematic review of the factors affecting accuracy of SUV measurements. AJR Am J Roentgenol United States. 2010;195:310-20.

24. Boellaard R. Standards for PET image acquisition and quantitative data analysis. J Nucl Med. 2009;50(Suppl 1):11S-20S.

25. Kinahan PE, Fletcher JW. Positron emission tomography-computed tomography standardized uptake values in clinical practice and assessing response to therapy. Semin Ultrasound CT MR United States. 2010;31:496-505. 
26. Fahey FH, Kinahan PE, Doot RK, Kocak M, Thurston H, Poussaint TY. Variability in PET quantitation within a multicenter consortium. Med Phys United States. 2010;37:3660-6.

27. Beyer T, Czernin J, Freudenberg LS. Variations in clinical PET/CT operations: results of an international survey of active PET/CT users. J Nucl Med United States. 2011;52:303-10.

28. Graham MM, Badawi RD, Wahl RL. Variations in PET/CT methodology for oncologic imaging at U.S. academic medical centers: an imaging response assessment team survey. J Nucl Med. 2011;52: 311-7.

29. Hristova I, Boellaard R, Vogel W, Mottaghy F, Marreaud S, Collette $\mathrm{S}$, et al. Retrospective quality control review of FDG scans in the imaging sub-study of PALETTE EORTC 62072/VEG110727: a randomized, double-blind, placebo-controlled phase III trial. Eur J Nucl Med Mol Imaging. 2015;42:848-57.

30. Boellaard R. Mutatis mutandis: harmonize the standard. J Nucl Med. 2012;53:1-3.

31. Buckler AJ, Boellaard R. Standardization of quantitative imaging: the time is right, and 18F-FDG PET/CT is a good place to start. J Nucl Med. 2011;52:171-2.

32. Sunderland JJ, Christian PE, Quantitative PET. CT scanner performance characterization based upon the Society of Nuclear Medicine and Molecular Imaging clinical trials network oncology clinical simulator phantom. J Nucl Med. 2015;56:145-52.

33. Rausch I, Cal-González J, Dapra D, Gallowitsch HJ, Lind P, Beyer $\mathrm{T}$, et al. Performance evaluation of the biograph $\mathrm{mCT}$ flow PET/CT system according to the NEMA NU2-2012 standard. EJNMMI Phys. 2015;2:26.

34. Lasnon C, Desmonts C, Quak E, Gervais R, Do P, Dubos-Arvis C, et al. Harmonizing SUVs in multicentre trials when using different generation PET systems: prospective validation in non-small cell lung cancer patients. Eur J Nucl Med Mol Imaging. 2013;40:98596.
35. Boellaard R, Delgado-Bolton R, Oyen WJG, Giammarile F, Tatsch $\mathrm{K}$, Eschner W, et al. FDG PET/CT: EANM procedure guidelines for tumour imaging: version 2.0. Eur J Nucl Med Mol Imaging. 2014;42:328-54.

36. Delbeke D, Coleman RE, Guiberteau MJ, Brown ML, Royal HD, Siegel BA, et al. Procedure guideline for tumor imaging with $18 \mathrm{~F}$ FDG PET/CT 1.0. J Nucl Med. 2006;47:885-95.

37. Graham MM, Wahl RL, Hoffman JM, Yap JT, Sunderland JJ, Boellaard $\mathrm{R}$, et al. Summary of the UPICT protocol for $18 \mathrm{~F}-\mathrm{FDG}$ PET/CT imaging in oncology clinical trials. J Nucl Med. 2015;56:955-61.

38. Makris NE, Huisman MC, Kinahan PE, Lammertsma AA, Boellaard R. Evaluation of strategies towards harmonization of FDG PET/CT studies in multicentre trials: comparison of scanner validation phantoms and data analysis procedures. Eur J Nucl Med Mol Imaging. 2013;40:1507-15.

39. Scheuermann JS, Saffer JR, Karp JS, Levering AM, Siegel A. Qualification of PET scanners for use in multicenter cancer clinical trials: the American College of Radiology Imaging Network experience. J Nucl Med. 2010;50:1187-93.

40. Kinahan P, Wahl R, Shao L, Frank R, Perlman E. The QIBA profile for quantitative FDG-PET/CT oncology imaging. J Nucl Med. 2014;55:1520.

41. Boellaard R, Willemsen AT, Arends B, Visser EP. EARL procedure for assessing PET/CT system specific patient FDG activity preparations for quantitative FDG PET/CT studies. 2013. p. 1-3. http:// earl.eanm.org/html/img/pool/EARL-procedure-for-optimizingFDGactivity-for-quantitative-FDG-PET-studies_version_1_1.pdf.

42. EANM EARL website [Internet]. Available from: http://earl.eanm.org.

43. Geworski L, Knoop BO, de Wit M, Ivancevic V, Bares R, Munz DL. Multicenter comparison of calibration and cross calibration of PET scanners. J Nucl Med. 2002;43:635-9. 